\title{
Fuzzy approximate solutions of second-order fuzzy linear boundary value problems
}

Xiaobin Guo ${ }^{1,3 *}$, Dequan Shang ${ }^{2}$ and Xiaoquan $\mathrm{Lu}^{3}$

\section{${ }^{*}$ Correspondence:}

guoxb@nwnu.edu.cn

${ }^{1}$ College of Mathematics and

Statistics, Northwest Normal

University, Lanzhou, 730070, China

${ }^{3}$ College of Chemistry and Chemical

Engineering, Northwest Normal University, Lanzhou, 730070, China

Full list of author information is

available at the end of the article

\begin{abstract}
In this paper, approximate solutions of second-order linear differential equations with fuzzy boundary conditions, in which coefficient functions maintain the sign, are investigated. The fuzzy linear boundary value problem is converted to a crisp function system of linear equations by the undetermined fuzzy coefficients method. The fuzzy approximate solution of the fuzzy linear differential equation is obtained by solving the crisp linear equations. Some numerical examples are given to illustrate the proposed method.
\end{abstract}

Keywords: fuzzy numbers; matrix analysis; fuzzy boundary value problems; fuzzy approximate solutions

\section{Introduction}

Nowadays, fuzzy differential equations (FDEs) is a popular topic studied by many researchers since it is utilized widely for the purpose of modeling problems in science and engineering. Most of the practical problems require the solution of a fuzzy differential equation (FDE) which satisfies fuzzy initial or boundary conditions, therefore a fuzzy initial or boundary problem should be solved. However, many fuzzy initial or boundary value problems could not be solved exactly, sometimes it is even impossible to find their analytical solutions. Thus, considering their approximate solutions is becoming more important.

Prior to discussing fuzzy differential equations and their associated numerical algorithms, it is necessary to present an appropriate brief introduction to derivative of the fuzzy-valued function. The concept of a fuzzy derivative was first introduced by Chang and Zadeh [1], followed up by Dubois and Prade [2] who used the extension principle in their approach. Other fuzzy derivative concepts were proposed by Puri and Ralescu [3] and Goetschel and Vaxman [4] as an extension of the Hukuhara derivative of multivalued functions. Kandel and Byatt $[5,6]$ applied the concept of fuzzy differential equation to the analysis of fuzzy dynamical problems.

The numerical methods for solving fuzzy differential equation are introduced in [7-9]. In 2001, Buckley and Feuring [10] presented two analytical methods for solving an $n$ thorder fuzzy linear differential equation with fuzzy initial conditions. Their first method of solution was to fuzzify the crisp solution and then check to see if it satisfies the fuzzy differential equations with fuzzy initial conditions. The second method was the reverse of the first method; in that they firstly solved the fuzzy initial value problem and then checked to see if it defined a fuzzy function. In 2008, Allahviranllo et al. [11] obtained the approximate solution of $n$ th-order linear differential equations with fuzzy initial conditions by

\section{焦 Springer}

(c) 2013 Guo et al.; licensee Springer. This is an Open Access article distributed under the terms of the Creative Commons Attribution License (http://creativecommons.org/licenses/by/2.0), which permits unrestricted use, distribution, and reproduction in any medium, provided the original work is properly cited. 
using the collocation method. In 2003, O'Regan et al. [12] proved a super-linear result for fuzzy boundary value problems relying on a general Schauder theorem in the metric space. Meanwhile Lakshmikantham et al. [13] investigated the solution of two-point boundary value problems associated with nonlinear fuzzy differential equations by using the extension principle. In 2008, Chen Minghao et al. [14] obtained the conclusion: twopoint boundary value problems have the analytic solution only on condition that the new structure and properties to the fuzzy number are given. But for second-order fuzzy linear boundary value problems

$$
y^{\prime \prime}+p(t) y^{\prime}+q(t) y=g(t), \quad t \in[a, b]
$$

associated with

$$
\begin{aligned}
& y(a)=\widetilde{\alpha}, \quad y(b)=\widetilde{\beta}, \\
& y^{\prime}(a)=\widetilde{\alpha}, \quad y^{\prime}(b)=\widetilde{\beta}, \\
& y^{\prime}(a)-\alpha_{0} y(a)=\widetilde{\alpha}, \quad y^{\prime}(b)+\beta_{0} y(b)=\widetilde{\beta},
\end{aligned}
$$

it is not the case. Once the coefficient functions $p(t), q(t), g(t)$ are continuous on $[a, b]$ and $p(t), q(t)$ maintain the sign on $[a, b]$, a unique solution must exist.

In this paper, we consider the approximate solution of a class of second-order linear differential Eq. (1.1) under fuzzy boundary value conditions (1.2), (1.3) and (1.4). Based on the undetermined fuzzy coefficients method, we convert a second-order linear differential equation to the crisp system of linear equations. Secondly, we investigate their cases according to indifferent cases of coefficient functions $p(t)$ and $q(t)$ from the original systems and build the corresponding crisp systems of linear equations. Then we derive a fuzzy approximate solution of the fuzzy linear differential equation from solving crisp function systems of linear equations. Finally, some examples are given to illustrate the proposed method. The structure of this paper is organized as follows.

In Section 2, we recall some basic definitions and results about fuzzy numbers as well as fuzzy derivative of the fuzzy-valued function. In Sections 3, 4 and 5, we build crisp function systems of linear equations via analyzing different cases based on the coefficient functions of the fuzzy linear differential equation in detail. The proposed algorithms are illustrated by solving some examples in Section 6 and the conclusion is drawn in Section 7 .

\section{Preliminaries}

\subsection{Fuzzy numbers}

Definition 2.1 [1] A fuzzy number is a fuzzy set like $u: R \rightarrow I=[0,1]$ which satisfies:

(1) $u$ is upper semi-continuous,

(2) $u(x)=0$ outside some interval $[c, d]$,

(3) there are real numbers $a, b$ such that $c \leq a \leq b \leq d$ and

(3.1) $u(x)$ is monotonic increasing on $[c, a]$,

(3.2) $u(x)$ is monotonic decreasing on $[b, d]$,

(3.3) $u(x)=1, a \leq x \leq b$. 
Let $E^{1}$ be the set of all real fuzzy numbers which are normal, upper semi-continuous, convex and compactly supported fuzzy sets.

Definition 2.2 [2] A fuzzy number $u$ in a parametric form is a pair $(\underline{u}, \bar{u})$ of functions $\underline{u}(r)$, $\bar{u}(r), 0 \leq r \leq 1$, which satisfies the following requirements:

(1) $\underline{u}(r)$ is a bounded monotonic increasing left continuous function,

(2) $\bar{u}(r)$ is a bounded monotonic decreasing left continuous function,

(3) $\underline{u}(r) \leq \bar{u}(r), 0 \leq r \leq 1$.

A crisp number $x$ is simply represented by $(\underline{u}(r), \bar{u}(r))=(x, x), 0 \leq r \leq 1$. By appropriate definitions, the fuzzy number space $\{(\underline{u}(r), \bar{u}(r))\}$ becomes a convex cone $E^{1}$ which could be embedded isomorphically and isometrically into a Banach space $[15,16]$.

Definition 2.3 [2] Let $x=(\underline{x}(r), \bar{x}(r)), y=(\underline{y}(r), \bar{y}(r)) \in E^{1}, 0 \leq r \leq 1$ and arbitrary $k \in R$. Then

(1) $x=y$ iff $\underline{x}(r)=y(r)$ and $\bar{x}(r)=\bar{y}(r)$,

(2) $x+y=(\underline{x}(r)+\underline{y}(r), \bar{x}(r)+\bar{y}(r))$

(3) $x-y=(\underline{x}(r)-\bar{y}(r), \bar{x}(r)-y(r))$,

(4) $k x= \begin{cases}(k x(r), k \bar{x}(r)), & k \geq 0, \\ (\bar{x} \bar{x}(r), k x(r)), & k<0 .\end{cases}$

Definition 2.4 [17] For arbitrary $u=(\underline{u}, \bar{u}), v=(\underline{v}, \bar{v}) \in E^{1}$, the quantity

$$
D(u, v)=\left(\int_{0}^{1}(\underline{u}(r)-\underline{v}(r))^{2} d r+\int_{0}^{1}(\bar{u}(r)-\bar{v}(r))^{2} d r\right)^{\frac{1}{2}}
$$

is the distance between fuzzy numbers $u$ and $v$.

\subsection{Second-order fuzzy boundary value problem}

Definition 2.5 [3] Let $x, y \in E^{1}$. If there exists $z \in E^{1}$ such that $x=y+z$, then $z$ is called the Hukuhara-difference of fuzzy numbers $x$ and $y$, and it is denoted by $z=x \ominus y$.

In this paper the $\ominus$ sign stands always for Hukuhara-difference, and let us remark that $x \ominus y \neq x+(-1) y$.

Definition 2.6 [18] Let $f:[a, b] \rightarrow E^{1}$ and $t_{0} \in[a, b]$. We say that $f$ is Hukuhara differential at $t_{0}$, if there exists an element $f^{\prime}\left(t_{0}\right) \in E^{1}$ such that for all $h>0$ sufficiently small, $\exists f\left(t_{0}+h\right) f\left(t_{0}\right), f\left(t_{0}\right) \ominus f\left(t_{0}-h\right)$ and the limits (in the metric D)

$$
\lim _{h \rightarrow 0} \frac{f\left(t_{0}+h\right) \ominus f\left(t_{0}\right)}{h}=\lim _{h \rightarrow 0} \frac{f\left(t_{0}\right) \ominus f\left(t_{0}-h\right)}{h}=f^{\prime}\left(t_{0}\right) .
$$

Lemma $2.1[19]$ If $g:[a, b] \rightarrow R$ is differential on $[a, b]$ such that $g^{\prime}, g^{\prime \prime}$ are nonnegative and monotonic increasing on $[a, b]$, then $\forall c \in E^{1}, f(x)=c \cdot g(x)$ is differential on $[a, b]$ and

$$
f^{\prime}(x)=c \cdot g^{\prime}(x), \quad f^{\prime \prime}(x)=c \cdot g^{\prime \prime}(x), \quad \forall x \in[a, b] .
$$

Definition 2.7 The second-order differential equation

$$
y^{\prime \prime}=f\left(t, y, y^{\prime}\right), \quad t \in[a, b]
$$


with the boundary value conditions

$$
\begin{aligned}
& y(a)=\widetilde{\alpha}, \quad y(b)=\widetilde{\beta}, \quad \widetilde{\alpha}, \widetilde{\beta} \in E^{1}, \\
& y^{\prime}(a)=\widetilde{\alpha}, \quad y^{\prime}(b)=\widetilde{\beta}, \quad \widetilde{\alpha}, \widetilde{\beta} \in E^{1}, \\
& y^{\prime}(a)-\alpha_{0} y(a)=\widetilde{\alpha}, \quad y^{\prime}(b)+\beta_{0} y(b)=\widetilde{\beta}, \quad \alpha_{0}, \beta_{0} \geq 0, \alpha_{0}+\beta_{0}>0,
\end{aligned}
$$

are called the second-order fuzzy boundary value problems (FBVPs). The differential Eq. (2.1) along with fuzzy boundary value conditions (2.2), (2.3) and (2.4) are said to be secondorder fuzzy differential equation No. 1, No. 2 and No. 3 boundary value problems, respectively.

In particular, when $f\left(t, y, y^{\prime}\right)$ is a linear function with respect to $y$ and $y^{\prime}$, Eq. (2.1) is reduced to Eq. (1.1)

$$
y^{\prime \prime}+p(t) y^{\prime}+q(t) y=g(t), \quad t \in[a, b],
$$

and it is a linear differential equation. In this paper, we discuss the approximate solution of the second-order fuzzy linear differential function boundary value problem. For simplicity, we only discuss the second-order fuzzy linear differential function with fuzzy boundary value conditions (2.3) and (2.4).

\section{Method for solving No. 2 FBVPs}

\subsection{The undetermined fuzzy coefficients method}

The undetermined fuzzy coefficients method is to seek an approximate solution as

$$
\tilde{y}_{N}(t)=\sum_{k=0}^{N} \widetilde{\theta}_{k} \phi_{k}(t),
$$

where $\phi_{k}(t), k=0,1, \ldots, N$, are positive basic functions whose all differentiations are positive. We compute the fuzzy coefficients in (3.1) by setting the error to zero as follows:

$$
E=D\left(\widetilde{y}^{\prime \prime}+p(t) \widetilde{y}^{\prime}+q(t) \widetilde{y}, \widetilde{g}(t)\right)+D\left(\widetilde{y}^{\prime}(a), \widetilde{\alpha}\right)+D\left(\widetilde{y}^{\prime}(b), \widetilde{\beta}\right) .
$$

Then we substitute (3.1) in (3.2) and represent them in parametric forms, then

$$
\left\{\begin{array}{l}
\frac{y^{\prime \prime}(t, r)+p(t) y^{\prime}(t, r)+q(t) y(t, r)}{y^{\prime}(a, r)=\underline{\alpha}(r),}=\underline{g(t, r),} \\
\overline{y^{\prime}(b, r)=\underline{\beta}(r),} \\
\overline{y^{\prime \prime}(t, r)+p(t) y^{\prime}(t, r)+q(t) y(t, r)}=\overline{g(t, r)} \\
\bar{y}^{\prime}(a, r)=\bar{\alpha}(r), \\
\bar{y}^{\prime}(b, r)=\bar{\beta}(r) .
\end{array}\right.
$$

Lemma 3.1 [11] Let basic functions $\phi_{k}(t), k=0,1, \ldots, N$, and all of their differentiations be positive, without loss of generality. Then $\left(\underline{y}_{N}\right)^{(i)}(t)=\underline{y_{N}^{(i)}}(t)$ and $\left(\bar{y}_{N}^{(i)}\right)(t)=\overline{y_{N}^{(i)}}(t), i=0,1,2$.

In order to solve Eq. (1.1) with condition (1.3), we need to investigate the system of Eq. (3.3). In this section we consider two cases. 


\subsection{Case $1 p(t) q(t) \geq 0$}

Suppose that coefficients $p(t), q(t)$ are nonnegative. From (3.3), we have

$$
\begin{aligned}
& \underline{y}^{\prime \prime}(t, r)+p(t) \underline{y^{\prime}}(t, r)+q(t) \underline{y}(t, r)=\underline{g}(t, r), \\
& \bar{y}^{\prime \prime}(t, r)+p(t) \bar{y}^{\prime}(t, r)+q(t) \bar{y}(t, r)=\bar{g}(t, r), \\
& \underline{y}^{\prime}(a, r)=\underline{\alpha}(r), \quad \underline{y}^{\prime}(b, r)=\underline{\beta}(r), \quad \bar{y}^{\prime}(a, r)=\bar{\alpha}(r), \quad \bar{y}^{\prime}(b, r)=\bar{\beta}(r) .
\end{aligned}
$$

And when coefficients $p(t), q(t)$ are negative, we have

$$
\begin{aligned}
& \underline{y}^{\prime \prime}(t, r)-p(t) \bar{y}^{\prime}(t, r)-q(t) \bar{y}(t, r)=\underline{g}(t, r), \\
& \bar{y}^{\prime \prime}(t, r)-p(t) \underline{y}^{\prime}(t, r)-q(t) \underline{y}(t, r)=\bar{g}(t, r) .
\end{aligned}
$$

If (3.1) is substituted in (3.4) and (3.5), respectively, then

$$
\sum_{k=0}^{N} \underline{\theta}_{k}(r) \phi_{k}^{\prime \prime}(t)+p(t) \sum_{k=0}^{N} \underline{\theta}_{k}(r) \phi_{k}^{\prime}(t)+q(t) \sum_{k=0}^{N} \underline{\theta}_{k}(r) \phi_{k}(t)=\underline{g}(t, r),
$$

and

$$
\sum_{k=0}^{N} \bar{\theta}_{k}(r) \phi_{k}^{\prime \prime}(t)+p(t) \sum_{k=0}^{N} \bar{\theta}_{k}(r) \phi_{k}^{\prime}(t)+q(t) \sum_{k=0}^{N} \bar{\theta}_{k}(r) \phi_{k}(t)=\bar{g}(t, r),
$$

also

$$
\begin{aligned}
& \sum_{k=0}^{N} \underline{\theta}_{k}(r) \phi_{k}^{\prime}(a)=\underline{\alpha}(r), \quad \sum_{k=0}^{N} \underline{\theta}_{k}(r) \phi_{k}^{\prime}(b)=\underline{\beta}(r), \\
& \sum_{k=0}^{N} \bar{\theta}_{k}(r) \phi_{k}^{\prime}(a)=\bar{\alpha}(r), \quad \sum_{k=0}^{N} \bar{\theta}_{k}(r) \phi_{k}^{\prime}(b)=\bar{\beta}(r) .
\end{aligned}
$$

By setting

$$
\left\{\begin{array}{l}
\gamma_{k}=\phi_{k}^{\prime \prime}(t)+p(t) \phi_{k}^{\prime}(t)+q(t) \phi_{k}(t), \quad k=0,1, \ldots, N, \\
\sigma_{a k}=\phi_{k}^{\prime}(a), \quad \sigma_{b k}=\phi_{k}^{\prime}(b),
\end{array}\right.
$$

the following system is obtained:

$$
\left\{\begin{array}{l}
\sum_{k=0}^{N} \underline{\theta}_{k}(r) \gamma_{k}=\underline{g}(t, r), \\
\sum_{k=0}^{N} \underline{\theta}_{k}(r) \sigma_{a k}=\underline{\alpha}(r), \\
\sum_{k=0}^{N} \underline{\theta}_{k}(r) \sigma_{b k}=\underline{\beta}(r) \\
\sum_{k=0}^{N} \bar{\theta}_{k}(r) \gamma_{k}=\bar{g}(t, r), \\
\sum_{k=0}^{N} \bar{\theta}_{k}(r) \sigma_{a k}=\bar{\alpha}(r), \\
\sum_{k=0}^{N} \bar{\theta}_{k}(r) \sigma_{b k}=\bar{\beta}(r) .
\end{array}\right.
$$

Equation (3.9) is a system of linear equations $S(t) X(r)=Y(r)$ such that

$$
S=\left(\begin{array}{ll}
S_{1} & S_{2} \\
S_{2} & S_{1}
\end{array}\right)
$$


where

$$
S_{1}=\left(\begin{array}{cccc}
\gamma_{0} & \gamma_{1} & \cdots & \gamma_{N} \\
\sigma_{a 0} & \sigma_{a 1} & \cdots & \sigma_{a N} \\
\sigma_{b 0} & \sigma_{b 1} & \cdots & \sigma_{b N}
\end{array}\right), \quad S_{2}=\left(\begin{array}{cccc}
0 & 0 & \cdots & 0 \\
0 & 0 & \cdots & 0 \\
0 & 0 & \cdots & 0
\end{array}\right) .
$$

And

$$
\begin{aligned}
& X=\left(\underline{\theta}_{0}, \underline{\theta}_{1}, \ldots, \underline{\theta}_{N}, \bar{\theta}_{0}, \bar{\theta}_{1}, \ldots, \bar{\theta}_{N}\right)^{\top}, \\
& Y=(\underline{g}(t, r), \underline{\alpha}(r), \underline{\beta}(r), \bar{g}(t, r), \bar{\alpha}(r), \bar{\beta}(r))^{\top} .
\end{aligned}
$$

The variables $\underline{\theta}_{0}, \underline{\theta}_{1}, \ldots, \underline{\theta}_{N}, \bar{\theta}_{0}, \bar{\theta}_{1}, \ldots, \bar{\theta}_{N}$ are obtained by solving (3.9) by setting $t=s, s \in$ $[a, b]$. These variables yield the fuzzy approximate solution $(y(t, r), \bar{y}(t, r))$.

In the same way, when coefficients $p(t), q(t)$ are negative, we build the corresponding system of linear equations as follows:

$$
\left(\begin{array}{cccccccc}
\gamma_{0} & \gamma_{1} & \cdots & \gamma_{N} & \delta_{0} & \delta_{1} & \cdots & \delta_{N} \\
\sigma_{a 0} & \sigma_{a 1} & \cdots & \sigma_{a N} & 0 & 0 & \cdots & 0 \\
\sigma_{b 0} & \sigma_{b 1} & \cdots & \sigma_{b N} & 0 & 0 & \cdots & 0 \\
\delta_{0} & \delta_{1} & \cdots & \delta_{N} & \gamma_{0} & \gamma_{1} & \cdots & \gamma_{N} \\
0 & 0 & \cdots & 0 & \sigma_{a 0} & \sigma_{a 1} & \cdots & \sigma_{a N} \\
0 & 0 & \cdots & 0 & \sigma_{b 0} & \sigma_{b 1} & \cdots & \sigma_{b N}
\end{array}\right)\left(\begin{array}{c}
\underline{\theta}_{0} \\
\underline{\theta}_{1} \\
\vdots \\
\underline{\theta}_{N} \\
\bar{\theta}_{0} \\
\bar{\theta}_{1} \\
\vdots \\
\bar{\theta}_{N}
\end{array}\right)=\left(\begin{array}{c}
\underline{g}(t, r) \\
\underline{\alpha}(r) \\
\underline{\beta}(r) \\
\bar{g}(t, r) \\
\bar{\alpha}(r) \\
\bar{\beta}(r)
\end{array}\right),
$$

where

$$
\left\{\begin{array}{l}
\gamma_{k}=\phi_{k}^{\prime \prime}(t), \\
\delta_{k}=-p(t) \phi_{k}^{\prime}(t)-q(t) \phi_{k}(t), \quad k=0,1, \ldots, N . \\
\sigma_{a k}=\phi_{k}^{\prime}(a), \quad \sigma_{b k}=\phi_{k}^{\prime}(b),
\end{array}\right.
$$

3.3 Case $2 p(t) q(t)<0$

Suppose that coefficient $p(t)$ is nonnegative and $q(t)$ is negative. From (3.3), we have

$$
\begin{aligned}
& \underline{y}^{\prime \prime}(t, r)+p(t) \underline{y}^{\prime}(t, r)-q(t) \bar{y}(t, r)=\underline{g}(t, r), \\
& \bar{y}^{\prime \prime}(t, r)+p(t) \bar{y}^{\prime}(t, r)-q(t) \underline{y}(t, r)=\bar{g}(t, r) .
\end{aligned}
$$

When coefficient $p(t)$ is negative and $q(t)$ is nonnegative, we have

$$
\begin{aligned}
& \underline{y}^{\prime \prime}(t, r)-p(t) \bar{y}^{\prime}(t, r)+q(t) \underline{y}(t, r)=\underline{g}(t, r), \\
& \bar{y}^{\prime \prime}(t, r)-p(t) \underline{y}^{\prime}(t, r)+q(t) \bar{y}(t, r)=\bar{g}(t, r) .
\end{aligned}
$$

If (3.1) is substituted in (3.11) and (3.12), respectively, then

$$
\sum_{k=0}^{N} \underline{\theta}_{k}(r) \phi_{k}^{\prime \prime}(t)+p(t) \sum_{k=0}^{N} \underline{\theta}_{k}(r) \phi_{k}^{\prime}(t)-q(t) \sum_{k=0}^{N} \bar{\theta}_{k}(r) \phi_{k}(t)=\underline{g}(t, r),
$$


and

$$
\sum_{k=0}^{N} \bar{\theta}_{k}(r) \phi_{k}^{\prime \prime}(t)+p(t) \sum_{k=0}^{N} \bar{\theta}_{k}(r) \phi_{k}^{\prime}(t)-q(t) \sum_{k=0}^{N} \underline{\theta}_{k}(r) \phi_{k}(t)=\bar{g}(t, r),
$$

also

$$
\begin{array}{lll}
\sum_{k=0}^{N} \underline{\theta}_{k}(r) \phi_{k}^{\prime}(a)=\underline{\alpha}(r), & & \sum_{k=0}^{N} \underline{\theta}_{k}(r) \phi_{k}^{\prime}(b)=\underline{\beta}(r), \\
\sum_{k=0}^{N} \bar{\theta}_{k}(r) \phi_{k}^{\prime}(a)=\bar{\alpha}(r), & & \sum_{k=0}^{N} \bar{\theta}_{k}(r) \phi_{k}^{\prime}(b)=\bar{\beta}(r) .
\end{array}
$$

By setting

$$
\left\{\begin{array}{l}
\zeta_{k}=\phi_{k}^{\prime \prime}(t)+p(t) \phi_{k}^{\prime}(t), \\
\xi_{k}=-q(t) \phi_{k}(t), \\
\sigma_{a k}=\phi_{k}^{\prime}(a), \quad \sigma_{b k}=\phi_{k}^{\prime}(b),
\end{array} \quad k=0,1, \ldots, N,\right.
$$

the following system is obtained:

$$
\left\{\begin{array}{l}
\sum_{k=0}^{N} \underline{\theta}_{k}(r) \zeta_{k}+\sum_{k=0}^{N} \bar{\theta}_{k}(r) \xi_{k}=\underline{g}(t, r), \\
\sum_{k=0}^{N} \underline{\theta}_{k}(r) \sigma_{a k}=\underline{\alpha}(r), \\
\sum_{k=0}^{N} \underline{\theta}_{k}(r) \sigma_{b k}=\underline{\beta}(r) \\
\sum_{k=0}^{N} \bar{\theta}_{k}(r) \zeta_{k}+\sum_{k=0}^{N} \underline{\theta}_{k}(r) \xi_{k}=\bar{g}(t, r), \\
\sum_{k=0}^{N} \bar{\theta}_{k}(r) \sigma_{a k}=\bar{\alpha}(r), \\
\sum_{k=0}^{N} \bar{\theta}_{k}(r) \sigma_{b k}=\bar{\beta}(r) .
\end{array}\right.
$$

Equation (3.15) is a system of linear equations $S(t) X(r)=Y(r)$ such that

$$
S=\left(\begin{array}{ll}
S_{1} & S_{2} \\
S_{2} & S_{1}
\end{array}\right)
$$

where

$$
S_{1}=\left(\begin{array}{cccc}
\zeta_{0} & \zeta_{1} & \cdots & \zeta_{N} \\
\sigma_{a 0} & \sigma_{a 1} & \cdots & \sigma_{a N} \\
\sigma_{b 0} & \sigma_{b 1} & \cdots & \sigma_{b N}
\end{array}\right), \quad S_{2}=\left(\begin{array}{cccc}
\xi_{0} & \xi_{1} & \cdots & \xi_{N} \\
0 & 0 & \cdots & 0 \\
0 & 0 & \cdots & 0
\end{array}\right)
$$

And

$$
\begin{aligned}
X & =\left(\underline{\theta}_{0}, \underline{\theta}_{1}, \ldots, \underline{\theta}_{N}, \bar{\theta}_{0}, \bar{\theta}_{1}, \ldots, \bar{\theta}_{N}\right)^{\top}, \\
Y & =(\underline{g}(t, r), \underline{\alpha}(r), \underline{\beta}(r), \bar{g}(t, r), \bar{\alpha}(r), \bar{\beta}(r))^{\top} .
\end{aligned}
$$

The variables $\underline{\theta}_{0}, \underline{\theta}_{1}, \ldots, \underline{\theta}_{N}, \bar{\theta}_{0}, \bar{\theta}_{1}, \ldots, \bar{\theta}_{N}$ are obtained by solving (3.15) by setting $t=s$, $s \in[a, b]$. These variables yield the fuzzy approximate solution $(\underline{y}(t, r), \bar{y}(t, r))$. 
In the same way, when coefficient $p(t)$ is negative and $q(t)$ is nonnegative, we set up the corresponding system of linear equations as follows:

$$
\left(\begin{array}{cccccccc}
\zeta_{0} & \zeta_{1} & \cdots & \zeta_{N} & \xi_{0} & \xi_{1} & \cdots & \xi_{N} \\
\sigma_{a 0} & \sigma_{a 1} & \cdots & \sigma_{a N} & 0 & 0 & \cdots & 0 \\
\sigma_{b 0} & \sigma_{b 1} & \cdots & \sigma_{b N} & 0 & 0 & \cdots & 0 \\
\xi_{0} & \xi_{1} & \cdots & \xi_{N} & \zeta_{0} & \zeta_{1} & \cdots & \zeta_{N} \\
0 & 0 & \cdots & 0 & \sigma_{a 0} & \sigma_{a 1} & \cdots & \sigma_{a N} \\
0 & 0 & \cdots & 0 & \sigma_{b 0} & \sigma_{b 1} & \cdots & \sigma_{b N}
\end{array}\right)\left(\begin{array}{c}
\underline{\theta}_{0} \\
\underline{\theta}_{1} \\
\vdots \\
\underline{\theta}_{N} \\
\bar{\theta}_{0} \\
\bar{\theta}_{1} \\
\vdots \\
\bar{\alpha}_{N}
\end{array}\right)=\left(\begin{array}{c}
\underline{g}(t, r) \\
\underline{\alpha}(r) \\
\underline{\beta}(r) \\
\bar{g}(t, r) \\
\bar{\alpha}(r) \\
\bar{\beta}(r)
\end{array}\right),
$$

where

$$
\left\{\begin{array}{l}
\zeta_{k}=\phi_{k}^{\prime \prime}(t)+q(t) \phi_{k}(t), \\
\xi_{k}=-p(t) \phi_{k}^{\prime}(t), \\
\sigma_{a k}=\phi_{k}^{\prime}(a), \quad \sigma_{b k}=\phi_{k}^{\prime}(b),
\end{array} \quad k=0,1, \ldots, N\right.
$$

\section{Method for solving No. 3 FBVPs}

\subsection{The undetermined fuzzy coefficients method}

Similarly, we compute the fuzzy coefficients in (3.1) by setting the error to zero as follows:

$$
E=D\left(\widetilde{y}^{\prime \prime}+p(t) \widetilde{y}^{\prime}+q(t) \widetilde{y}, \widetilde{g}(t)\right)+D\left(\widetilde{y}^{\prime}(a)-\alpha_{0} \widetilde{y}(a), \widetilde{\alpha}\right)+D\left(\widetilde{y}^{\prime}(b)+\beta_{0} \widetilde{y}(b), \widetilde{\beta}\right) .
$$

Then we substitute (3.1) in (4.1) and represent them in parametric forms:

$$
\left\{\begin{array}{l}
\frac{y^{\prime \prime}(t, r)+p(t) y^{\prime}(t, r)+q(t) y(t, r)}{y^{\prime}(a, r)+\alpha_{0} \bar{y}(a, r)=\underline{\alpha}(r),}=\underline{g(t, r),} \\
\overline{y^{\prime}}(b, r)+\beta_{0} \underline{y}(b, r)=\underline{\beta}(r), \\
\overline{y^{\prime \prime}(t, r)+p(t) y^{\prime}(t, r)+q(t) y(t, r)}=\overline{g(t, r)}, \\
\bar{y}^{\prime}(a, r)+\alpha_{0} \underline{y}(a, r)=\bar{\alpha}(r), \\
\bar{y}^{\prime}(b, r)+\beta_{0} \overline{\bar{y}}(b, r)=\bar{\beta}(r) .
\end{array}\right.
$$

In order to solve Eq. (1.1) with Eq. (1.4), we need to investigate the system of Eq. (4.3). In this section we also consider two cases.

\subsection{Case $1 p(t) q(t) \geq 0$}

Suppose that coefficients $p(t), q(t)$ are nonnegative. From (4.2), we have

$$
\begin{aligned}
& \underline{y}^{\prime \prime}(t, r)+p(t) \underline{y}^{\prime}(t, r)+q(t) \underline{y}(t, r)=\underline{g}(t, r), \\
& \bar{y}^{\prime \prime}(t, r)+p(t) \bar{y}^{\prime}(t, r)+q(t) \bar{y}(t, r)=\bar{g}(t, r), \\
& \underline{y}^{\prime}(a, r)+\alpha_{0} \bar{y}(a, r)=\underline{\alpha}(r), \quad \underline{y}^{\prime}(b, r)+\beta_{0} \underline{y}(b, r)=\underline{\beta}(r), \\
& \bar{y}^{\prime}(a, r)+\alpha_{0} \underline{y}(a, r)=\bar{\alpha}(r), \quad \bar{y}^{\prime}(b, r)+\beta_{0} \bar{y}(b, r)=\bar{\beta}(r) .
\end{aligned}
$$


And when coefficients $p(t), q(t)$ are negative, we have

$$
\begin{aligned}
& \underline{y}^{\prime \prime}(t, r)-p(t) \bar{y}^{\prime}(t, r)-q(t) \bar{y}(t, r)=\underline{g}(t, r), \\
& \bar{y}^{\prime \prime}(t, r)-p(t) \underline{y^{\prime}}(t, r)-q(t) \underline{y}(t, r)=\bar{g}(t, r) .
\end{aligned}
$$

If (3.1) is substituted in (4.3) and (4.4), respectively, then

$$
\sum_{k=0}^{N} \underline{\theta}_{k}(r) \phi_{k}^{\prime \prime}(t)+p(t) \sum_{k=0}^{N} \underline{\theta}_{k}(r) \phi_{k}^{\prime}(t)+q(t) \sum_{k=0}^{N} \underline{\theta}_{k}(r) \phi_{k}(t)=\underline{g}(t, r),
$$

and

$$
\sum_{k=0}^{N} \bar{\theta}_{k}(r) \phi_{k}^{\prime \prime}(t)+p(t) \sum_{k=0}^{N} \bar{\theta}_{k}(r) \phi_{k}^{\prime}(t)+q(t) \sum_{k=0}^{N} \bar{\theta}_{k}(r) \phi_{k}(t)=\bar{g}(t, r)
$$

also

$$
\begin{aligned}
& \sum_{k=0}^{N} \underline{\theta}_{k}(r) \phi_{k}^{\prime}(a)+\alpha_{0} \sum_{k=0}^{N} \bar{\theta}_{k}(r) \phi_{k}(a)=\underline{\alpha}(r), \\
& \sum_{k=0}^{N} \underline{\theta}_{k}(r) \phi_{k}^{\prime}(b)+\beta_{0} \sum_{k=0}^{N} \underline{\theta}_{k}(r) \phi_{k}(b)=\underline{\beta}(r), \\
& \sum_{k=0}^{N} \bar{\theta}_{k}(r) \phi_{k}^{\prime}(a)+\alpha_{0} \sum_{k=0}^{N} \underline{\theta}_{k}(r) \phi_{k}(a)=\bar{\alpha}(r), \\
& \sum_{k=0}^{N} \bar{\theta}_{k}(r) \phi_{k}^{\prime}(b)+\beta_{0} \sum_{k=0}^{N} \bar{\theta}_{k}(r) \phi_{k}(b)=\bar{\beta}(r) .
\end{aligned}
$$

By setting

$$
\left\{\begin{array}{l}
\gamma_{k}=\phi_{k}^{\prime \prime}(t)+p(t) \phi_{k}^{\prime}(t)+q(t) \phi_{k}(t), \\
\sigma_{a k}=\phi_{k}^{\prime}(a), \sigma_{b k}=\alpha_{0} \phi_{k}(a), \\
\delta(k)=\phi_{k}^{\prime}(b)+\beta_{0} \phi_{k}(b),
\end{array} \quad k=0,1, \ldots, N\right.
$$

the following system is obtained:

$$
\left\{\begin{array}{l}
\sum_{k=0}^{N} \underline{\theta}_{k}(r) \gamma_{k}=\underline{g}(t, r), \\
\sum_{k=0}^{N} \underline{\theta}_{k}(r) \sigma_{a k}+\sum_{k=0}^{N} \bar{\theta}_{k}(r) \sigma_{b k}=\underline{\alpha}(r), \\
\sum_{k=0}^{N} \underline{\theta}_{k}(r) \delta_{k}=\underline{\beta}(r) \\
\sum_{k=0}^{N} \bar{\theta}_{k}(r) \gamma_{k}=\overline{\bar{g}}(t, r), \\
\sum_{k=0}^{N} \bar{\theta}_{k}(r) \sigma_{a k}+\sum_{k=0}^{N} \underline{\theta}_{k}(r) \sigma_{b k}=\bar{\alpha}(r), \\
\sum_{k=0}^{N} \bar{\theta}_{k}(r) \delta_{k}=\bar{\beta}(r) .
\end{array}\right.
$$

Equation (4.9) is a system of linear equations $S(t) X(r)=Y(r)$ such that

$$
S=\left(\begin{array}{ll}
S_{1} & S_{2} \\
S_{2} & S_{1}
\end{array}\right)
$$


where

$$
S_{1}=\left(\begin{array}{cccc}
\gamma_{0} & \gamma_{1} & \cdots & \gamma_{N} \\
\sigma_{a 0} & \sigma_{a 1} & \cdots & \sigma_{a N} \\
\delta_{b 0} & \delta_{b 1} & \cdots & \delta_{b N}
\end{array}\right), \quad S_{2}=\left(\begin{array}{cccc}
0 & 0 & \cdots & 0 \\
\sigma_{b 0} & \sigma_{b 1} & \cdots & \sigma_{b N} \\
0 & 0 & \cdots & 0
\end{array}\right)
$$

And

$$
\begin{aligned}
& X=\left(\underline{\theta}_{0}, \underline{\theta}_{1}, \ldots, \underline{\theta}_{N}, \bar{\theta}_{0}, \bar{\theta}_{1}, \ldots, \bar{\theta}_{N}\right)^{\top}, \\
& Y=(\underline{g}(t, r), \underline{\alpha}(r), \underline{\beta}(r), \bar{g}(t, r), \bar{\alpha}(r), \bar{\beta}(r))^{\top} .
\end{aligned}
$$

The variables $\underline{\theta}_{0}, \underline{\theta}_{1}, \ldots, \underline{\theta}_{N}, \bar{\theta}_{0}, \bar{\theta}_{1}, \ldots, \bar{\theta}_{N}$ are obtained by solving (4.9) by setting $t=s, s \in$ $[a, b]$. These variables yield the fuzzy approximate solution $(y(t, r), \bar{y}(t, r))$.

Similarly, when coefficients $p(t), q(t)$ are negative, we build the corresponding system of linear equations as follows:

$$
\left(\begin{array}{cccccccc}
\gamma_{0} & \gamma_{1} & \cdots & \gamma_{N} & \eta_{0} & \eta_{1} & \cdots & \eta_{N} \\
\sigma_{a 0} & \sigma_{a 1} & \cdots & \sigma_{a N} & \sigma_{b 0} & \sigma_{b 1} & \cdots & \sigma_{b N} \\
\delta_{0} & \delta_{1} & \cdots & \delta_{N} & 0 & 0 & \cdots & 0 \\
\eta_{0} & \eta_{1} & \cdots & \eta_{N} & \gamma_{0} & \gamma_{1} & \cdots & \gamma_{N} \\
\sigma_{b 0} & \sigma_{b 1} & \cdots & \sigma_{b N} & \sigma_{a 0} & \sigma_{a 1} & \cdots & \sigma_{a N} \\
0 & 0 & \cdots & 0 & \delta_{0} & \delta_{1} & \cdots & \delta_{N}
\end{array}\right)\left(\begin{array}{c}
\underline{\theta}_{0} \\
\underline{\theta}_{1} \\
\vdots \\
\underline{\theta}_{N} \\
\bar{\theta}_{0} \\
\bar{\theta}_{1} \\
\vdots \\
\bar{\theta}_{N}
\end{array}\right)=\left(\begin{array}{c}
\underline{g}(t, r) \\
\underline{\alpha}(r) \\
\underline{\beta}(r) \\
\bar{g}(t, r) \\
\bar{\alpha}(r) \\
\bar{\beta}(r)
\end{array}\right),
$$

where

$$
\left\{\begin{array}{l}
\gamma_{k}=\phi_{k}^{\prime \prime}(t), \quad \eta_{k}=-p(t) \phi_{k}^{\prime}(t)-q(t) \phi_{k}(t), \\
\sigma_{a k}=\phi_{k}^{\prime}(a), \quad \sigma_{b k}=\alpha_{0} \phi_{k}(a), \\
\delta_{(}(k)=\phi_{k}^{\prime}(b)+\beta_{0} \phi_{k}(b),
\end{array} \quad k=0,1, \ldots, N,\right.
$$

4.3 Case $2 p(t) q(t)<0$

Suppose that coefficient $p(t)$ is nonnegative and $q(t)$ is negative. From (4.3), we have

$$
\begin{aligned}
& \underline{y}^{\prime \prime}(t, r)+p(t) \underline{y}^{\prime}(t, r)-q(t) \bar{y}(t, r)=\underline{g}(t, r), \\
& \bar{y}^{\prime \prime}(t, r)+p(t) \bar{y}^{\prime}(t, r)-q(t) \underline{y}(t, r)=\bar{g}(t, r) .
\end{aligned}
$$

When coefficient $p(t)$ is negative and $q(t)$ is nonnegative, we have

$$
\begin{aligned}
& \underline{y}^{\prime \prime}(t, r)-p(t) \bar{y}^{\prime}(t, r)+q(t) \underline{y}(t, r)=\underline{g}(t, r), \\
& \bar{y}^{\prime \prime}(t, r)-p(t) \underline{y}^{\prime}(t, r)+q(t) \bar{y}(t, r)=\bar{g}(t, r) .
\end{aligned}
$$

If (3.1) is substituted in (4.11) and (4.12), respectively, then

$$
\sum_{k=0}^{N} \underline{\theta}_{k}(r) \phi_{k}^{\prime \prime}(t)-p(t) \sum_{k=0}^{N} \underline{\theta}_{k}(r) \phi_{k}^{\prime}(t)+q(t) \sum_{k=0}^{N} \bar{\theta}_{k}(r) \phi_{k}(t)=\underline{g}(t, r),
$$


and

$$
\sum_{k=0}^{N} \bar{\theta}_{k}(r) \phi_{k}^{\prime \prime}(t)-p(t) \sum_{k=0}^{N} \bar{\theta}_{k}(r) \phi_{k}^{\prime}(t)+q(t) \sum_{k=0}^{N} \underline{\theta}_{k}(r) \phi_{k}(t)=\bar{g}(t, r)
$$

also

$$
\begin{aligned}
& \sum_{k=0}^{N} \underline{\theta}_{k}(r) \phi_{k}^{\prime}(a)+\alpha_{0} \sum_{k=0}^{N} \bar{\theta}_{k}(r) \phi_{k}(a)=\underline{\alpha}(r), \\
& \sum_{k=0}^{N} \underline{\theta}_{k}(r) \phi_{k}^{\prime}(b)+\beta_{0} \sum_{k=0}^{N} \underline{\theta}_{k}(r) \phi_{k}(b)=\underline{\beta}(r), \\
& \sum_{k=0}^{N} \bar{\theta}_{k}(r) \phi_{k}^{\prime}(a)+\alpha_{0} \sum_{k=0}^{N} \underline{\theta}_{k}(r) \phi_{k}(a)=\bar{\alpha}(r), \\
& \sum_{k=0}^{N} \bar{\theta}_{k}(r) \phi_{k}^{\prime}(b)+\beta_{0} \sum_{k=0}^{N} \bar{\theta}_{k}(r) \phi_{k}(b)=\bar{\beta}(r) .
\end{aligned}
$$

By setting

$$
\left\{\begin{array}{l}
\gamma_{k}=\phi_{k}^{\prime \prime}(t)+p(t) \phi_{k}^{\prime}(t), \quad \xi_{k}=-q(t) \phi_{k}(t), \\
\sigma_{a k}=\phi_{k}^{\prime}(a), \quad \sigma_{b k}=\alpha_{0} \phi_{k}(a), \\
\delta(k)=\phi_{k}^{\prime}(b)+\beta_{0} \phi_{k}(b),
\end{array} \quad k=0,1, \ldots, N\right.
$$

the following system is obtained:

$$
\left\{\begin{array}{l}
\sum_{k=0}^{N} \underline{\theta}_{k}(r) \gamma_{k}+\sum_{k=0}^{N} \bar{\theta}_{k}(r) \xi_{k}=g(t, r), \\
\sum_{k=0}^{N} \underline{\theta}_{k}(r) \sigma_{a k}+\sum_{k=0}^{N} \bar{\theta}_{k}(r) \sigma_{b k}=\underline{\alpha}(r), \\
\sum_{k=0}^{N} \underline{\theta}_{k}(r) \delta_{k}=\underline{\beta}(r), \\
\sum_{k=0}^{N} \bar{\theta}_{k}(r) \gamma_{k}+\sum_{k=0}^{N} \underline{\theta}_{k}(r) \xi_{k}=\bar{g}(t, r), \\
\sum_{k=0}^{N} \bar{\theta}_{k}(r) \sigma_{a k}+\sum_{k=0}^{N} \underline{\theta}_{k}(r) \sigma_{b k}=\bar{\alpha}(r), \\
\sum_{k=0}^{N} \bar{\theta}_{k}(r) \delta_{k}=\bar{\beta}(r) .
\end{array}\right.
$$

Equation (4.15) is a system of linear equations $S(t) X(t)=Y(r)$ such that

$$
S=\left(\begin{array}{ll}
S_{1} & S_{2} \\
S_{2} & S_{1}
\end{array}\right)
$$

where

$$
S_{1}=\left(\begin{array}{cccc}
\gamma_{0} & \gamma_{1} & \cdots & \gamma_{N} \\
\sigma_{a 0} & \sigma_{a 1} & \cdots & \sigma_{a N} \\
\delta_{0} & \delta_{1} & \cdots & \delta_{N}
\end{array}\right), \quad S_{2}=\left(\begin{array}{cccc}
\xi_{0} & \xi_{1} & \cdots & \xi_{N} \\
\sigma_{b 0} & \sigma_{b 1} & \cdots & \sigma_{b N} \\
0 & 0 & \cdots & 0
\end{array}\right)
$$

And

$$
\begin{aligned}
X & =\left(\underline{\theta}_{0}, \underline{\theta}_{1}, \ldots, \underline{\theta}_{N}, \bar{\theta}_{0}, \bar{\theta}_{1}, \ldots, \bar{\theta}_{N}\right)^{\top}, \\
Y & =(\underline{g}(t, r), \underline{\alpha}(r), \underline{\beta}(r), \bar{g}(t, r), \bar{\alpha}(r), \bar{\beta}(r))^{\top} .
\end{aligned}
$$


The variables $\underline{\theta}_{0}, \underline{\theta}_{1}, \ldots, \underline{\theta}_{N}, \bar{\theta}_{0}, \bar{\theta}_{1}, \ldots, \bar{\theta}_{N}$ are obtained by solving (4.14) by setting $t=s$, $s \in[a, b]$. These variables yield the fuzzy approximate solution $(\underline{y}(t, r), \bar{y}(t, r))$.

Similarly, when coefficient $p(t)$ is negative and $q(t)$ is nonnegative, we extend the corresponding system of linear equations as follows:

$$
\left(\begin{array}{cccccccc}
\gamma_{0} & \gamma_{1} & \cdots & \gamma_{N} & \xi_{0} & \xi_{1} & \cdots & \xi_{N} \\
\sigma_{a 0} & \sigma_{a 1} & \cdots & \sigma_{a N} & \sigma_{b 0} & \sigma_{b 1} & \cdots & \sigma_{b N} \\
\delta_{0} & \delta_{1} & \cdots & \delta_{N} & 0 & 0 & \cdots & 0 \\
\xi_{0} & \xi_{1} & \cdots & \xi_{N} & \gamma_{0} & \gamma_{1} & \cdots & \gamma_{N} \\
\sigma_{b 0} & \sigma_{b 1} & \cdots & \sigma_{b N} & \sigma_{a 0} & \sigma_{a 1} & \cdots & \sigma_{a N} \\
0 & 0 & \cdots & 0 & \delta_{0} & \delta_{1} & \cdots & \delta_{N}
\end{array}\right)\left(\begin{array}{c}
\underline{\theta}_{0} \\
\underline{\theta}_{1} \\
\vdots \\
\underline{\theta}_{N} \\
\bar{\theta}_{0} \\
\bar{\theta}_{1} \\
\vdots \\
\bar{\theta}_{N}
\end{array}\right)=\left(\begin{array}{c}
\underline{g}(t, r) \\
\underline{\alpha}(r) \\
\underline{\beta}(r) \\
\bar{g}(t, r) \\
\bar{\alpha}(r) \\
\bar{\beta}(r)
\end{array}\right),
$$

where

$$
\left\{\begin{array}{l}
\gamma_{k}=\phi_{k}^{\prime \prime}(t)+q(t) \phi_{k}(t), \quad \xi_{k}=-p(t) \phi_{k}^{\prime}(t), \\
\sigma_{a k}=\phi_{k}^{\prime}(a), \quad \sigma_{b k}=\alpha_{0} \phi_{k}(a), \\
\delta_{(}(k)=\phi_{k}^{\prime}(b)+\beta_{0} \phi_{k}(b),
\end{array} \quad k=0,1, \ldots, N .\right.
$$

Likewise, for Eq. (1.1) with fuzzy boundary conditions (1.2), the following results are obvious.

\section{Approximate solutions of second-order FLBVPs}

The above model linear Eqs. (3.9), (3.10), (3.15), (3.16), (4.9), (4.10), (4.15) and (4.16) are $6 \times 2(N+1)$ function systems of linear equations and they have the same form as follows:

$$
S(t) X(r)=Y(r) \text {. }
$$

In the process of solving Eq. (5.1) by setting $t=q, q \in[a, b]$, no matter whether it is consistent or inconsistent, we obtain the minimal norm least squares solution [20] by using the generalized inverse of the coefficient matrix $S$, i.e.,

$$
X(r)=S^{\dagger}(q) Y(r)
$$

Thus we get

$$
\underline{\theta}_{0}, \underline{\theta}_{1}, \ldots, \underline{\theta}_{N}, \bar{\theta}_{0}, \bar{\theta}_{1}, \ldots, \bar{\theta}_{N} \text {. }
$$

Therefore, we obtain the fuzzy approximate solution of the original fuzzy linear differential equation as follows:

$$
\begin{aligned}
& \underline{y}(t, r)=\underline{\theta}_{0}(r) \phi_{0}(t)+\underline{\theta}_{1}(r) \phi_{1}(t)+\cdots+\underline{\theta}_{N}(r) \phi_{N}(t), \\
& \bar{y}(t, r)=\bar{\theta}_{0}(r) \phi_{0}(t)+\bar{\theta}_{1}(r) \phi_{1}(t)+\cdots+\bar{\theta}_{N}(r) \phi_{N}(t) .
\end{aligned}
$$




\section{Numerical examples}

Example 6.1 Consider the following second-order fuzzy linear differential equation:

$$
\left\{\begin{array}{l}
y^{\prime \prime}+y=-t, \quad t \in\left[0, \frac{\pi}{2}\right] \\
\widetilde{y}(0)=(-0.1+0.1 r, 0.1-0.1 r), \\
\widetilde{y}\left(\frac{\pi}{2}\right)=\left(-\frac{\pi}{2}+0.1 r, 1+\frac{\pi}{2}-0.1 r\right) .
\end{array}\right.
$$

The exact solution is as follows:

$$
\begin{aligned}
& \underline{Y}(t, r)=(-0.1+0.1 r) \cos t+(0.1 r) \sin t-t, \\
& \bar{Y}(t, r)=(0.1-0.1 r) \cos t+(1+\pi-0.1 r) \sin t-t .
\end{aligned}
$$

If $\phi_{k}(t)=t^{k}, k=0,1,2,3$, then

$$
\underline{y}(t, r)=\underline{\alpha}_{0}+\underline{\alpha}_{1} t+\underline{\alpha}_{2} t^{2}+\underline{\alpha}_{3} t^{3}, \quad \bar{y}(t, r)=\bar{\alpha}_{0}+\bar{\alpha}_{1} t+\bar{\alpha}_{2} t^{2}+\bar{\alpha}_{3} t^{3} .
$$

From (3.11), we build the following system:

$$
\left(\begin{array}{cccccccc}
1 & t & 2+t^{2} & 6 t+t^{3} & 0 & 0 & 0 & 0 \\
1 & 0 & 0 & 0 & 0 & 0 & 0 & 0 \\
0 & 1 & 0 & 0 & 0 & 0 & 0 & 0 \\
0 & 0 & 0 & 0 & 1 & t & 2+t^{2} & 6 t+t^{3} \\
0 & 0 & 0 & 0 & 1 & 0 & 0 & 0 \\
0 & 0 & 0 & 0 & 0 & 1 & 0 & 0
\end{array}\right)\left(\begin{array}{l}
\underline{\theta}_{0}(r) \\
\underline{\theta}_{1}(r) \\
\underline{\theta}_{2}(r) \\
\underline{\theta}_{3}(r) \\
\bar{\theta}_{0}(r) \\
\bar{\theta}_{1}(r) \\
\bar{\theta}_{2}(r) \\
\bar{\theta}_{3}(r)
\end{array}\right)=\left(\begin{array}{c}
-t \\
-0.1+0.1 r \\
-\frac{\pi}{2}+0.1 r \\
-t \\
0.1-0.1 r \\
1+\frac{\pi}{2}-0.1 r
\end{array}\right) .
$$

By setting $t=\frac{1}{3}$, the parameters $\underline{\alpha}_{0}, \underline{\alpha}_{1}, \underline{\alpha}_{2}, \underline{\alpha}_{3}, \bar{\alpha}_{0}, \bar{\alpha}_{1}, \bar{\alpha}_{2}, \bar{\alpha}_{3}$ are obtained, and by putting them into (5.3), we have

$$
\begin{aligned}
\underline{y}(t, r)= & (-0.100+0.100 r)+(1.5708+0.100 r) t \\
& +(-0.0674-0.0229 r) t^{2}+(-0.0936-0.03165 r) t^{3}, \\
\bar{y}(t, r)= & (0.100-0.100 r)+(4.142-0.100 r) t \\
& +(-0.1128+0.0227 r) t^{2}+(-0.1568+0.03161 r) t^{3} .
\end{aligned}
$$

Tables 1, 2, 3 and 4 show the comparisons between the exact solution and the approximate solution at $t=0.01$ and $t=0.001$ for some $r \in[0,1]$; all data are computed by Matlab7.x.

Example 6.2 Consider the following second-order fuzzy linear differential equation:

$$
\left\{\begin{array}{l}
y^{\prime \prime}-4 y^{\prime}+4 y=4 t-4, \quad t \geq 0 \\
\tilde{y}^{\prime}(0)=(3+2 r, 6-5 r), \\
\tilde{y}^{\prime}(1)=\left((-1+5 r) e^{2}+1,(7-5 r) e^{2}+1\right) .
\end{array}\right.
$$


Table 1 Comparisons between the exact solution and the approximate solution

\begin{tabular}{llll}
\hline $\boldsymbol{r}$ & $\underline{\boldsymbol{r}(\boldsymbol{t}, \boldsymbol{r})}$ & $\underline{\underline{y}(\boldsymbol{t}, \boldsymbol{r})}$ & Error \\
\hline 0 & -0.09911518137409 & -0.09912683067200 & $0.11649297906799 \mathrm{e}-4$ \\
0.1 & -0.08901568303658 & -0.08902706143300 & $0.11378396415129 \mathrm{e}-4$ \\
0.2 & -0.07891618469908 & -0.07892729219400 & $0.11107494923460 \mathrm{e}-4$ \\
0.3 & -0.06881668636157 & -0.06882752295500 & $0.10836593431776 \mathrm{e}-4$ \\
0.4 & -0.05871718802406 & -0.05872775371600 & $0.10565691940079 \mathrm{e}-4$ \\
0.5 & -0.04861768968655 & -0.04862798447700 & $0.10294790448395 \mathrm{e}-4$ \\
0.6 & -0.03851819134904 & -0.03852821523800 & $0.10023888956719 \mathrm{e}-4$ \\
0.7 & -0.02841869301153 & -0.02842844599900 & $0.09752987465039 \mathrm{e}-4$ \\
0.8 & -0.01831919467403 & -0.01832867676000 & $0.09482085973359 \mathrm{e}-4$ \\
0.9 & -0.00821969633652 & -0.00822890752100 & $0.09211184481680 \mathrm{e}-4$ \\
1 & 0.00187980200099 & 0.00187086171800 & $0.08940282990000 \mathrm{e}-4$ \\
\hline
\end{tabular}

Table 2 Comparisons between the exact solution and the approximate solution

\begin{tabular}{llll}
\hline $\boldsymbol{r}$ & $\overline{\boldsymbol{Y}}(\boldsymbol{t}, \boldsymbol{r})$ & $\overline{\mathbf{y}}(\boldsymbol{t}, \boldsymbol{r})$ & Error \\
\hline 0 & 0.10287478537607 & 0.10286855421000 & $0.62311660731923 \mathrm{e}-5$ \\
0.1 & 0.09277528703856 & 0.09276878497120 & $0.65020673648691 \mathrm{e}-5$ \\
0.2 & 0.08267578870106 & 0.08266901573240 & $0.67729686565599 \mathrm{e}-5$ \\
0.3 & 0.07257629036355 & 0.07256924649360 & $0.70438699482367 \mathrm{e}-5$ \\
0.4 & 0.06247679202604 & 0.06246947725480 & $0.73147712399066 \mathrm{e}-5$ \\
0.5 & 0.05237729368853 & 0.05236970801600 & $0.75856725315904 \mathrm{e}-5$ \\
0.6 & 0.04227779535102 & 0.04226993877720 & $0.78565738232741 \mathrm{e}-5$ \\
0.7 & 0.03217829701351 & 0.03217016953840 & $0.81274751149579 \mathrm{e}-5$ \\
0.8 & 0.02207879867601 & 0.02207040029960 & $0.83983764066348 \mathrm{e}-5$ \\
0.9 & 0.01197930033850 & 0.01197063106080 & $0.86692776983185 \mathrm{e}-5$ \\
1 & 0.00187980200099 & 0.00187086182200 & $0.89401789899965 \mathrm{e}-5$ \\
\hline
\end{tabular}

Table 3 Comparisons between the exact solution and the approximate solution

\begin{tabular}{llll}
\hline $\boldsymbol{r}$ & $\underline{\boldsymbol{r}(\boldsymbol{t}, \boldsymbol{r})}$ & $\underline{\boldsymbol{y}(\boldsymbol{t}, \boldsymbol{r})}$ & Error \\
\hline 0 & -0.09991195018134 & -0.09991206746457 & $0.11728323451310 \mathrm{e}-6$ \\
0.1 & -0.08990195518300 & -0.08990206974373 & $0.11456072925020 \mathrm{e}-6$ \\
0.2 & -0.07989196018467 & -0.07989207202289 & $0.11183822400118 \mathrm{e}-6$ \\
0.3 & -0.06988196518634 & -0.06988207430206 & $0.10911571875216 \mathrm{e}-6$ \\
0.4 & -0.05987197018800 & -0.05987207658122 & $0.10639321350314 \mathrm{e}-6$ \\
0.5 & -0.04986197518967 & -0.04986207886038 & $0.10367070826106 \mathrm{e}-6$ \\
0.6 & -0.03985198019133 & -0.03985208113954 & $0.10094820300510 \mathrm{e}-6$ \\
0.7 & -0.02984198519300 & -0.02984208341870 & $0.09822569775955 \mathrm{e}-6$ \\
0.8 & -0.01983199019467 & -0.01983208569786 & $0.09550319251053 \mathrm{e}-6$ \\
0.9 & -0.00982199519633 & -0.00982208797702 & $0.09278068726151 \mathrm{e}-6$ \\
1 & 0.00018799980200 & 0.00018790974382 & $0.09005818201011 \mathrm{e}-6$ \\
\hline
\end{tabular}

Table 4 Comparisons between the exact solution and the approximate solution

\begin{tabular}{llll}
\hline $\boldsymbol{r}$ & $\overline{\boldsymbol{Y}}(\boldsymbol{t}, \boldsymbol{r})$ & $\overline{\boldsymbol{y}}(\boldsymbol{t}, \boldsymbol{r})$ & Error \\
\hline 0 & 0.10028794978534 & 0.10028788695321 & $0.62832127500911 \mathrm{e}-7$ \\
0.1 & 0.09027795478700 & 0.09027788923237 & $0.65554632569520 \mathrm{e}-7$ \\
0.2 & 0.08026795978867 & 0.08026789151153 & $0.68277137610373 \mathrm{e}-7$ \\
0.3 & 0.07025796479034 & 0.07025789379069 & $0.70999642678982 \mathrm{e}-7$ \\
0.4 & 0.06024796979200 & 0.06024789606985 & $0.73722147712896 \mathrm{e}-7$ \\
0.5 & 0.05023797479367 & 0.05023789834902 & $0.76444652760688 \mathrm{e}-7$ \\
0.6 & 0.04022797979534 & 0.04022790062818 & $0.79167157815418 \mathrm{e}-7$ \\
0.7 & 0.03021798479700 & 0.03021790290734 & $0.81889662859741 \mathrm{e}-7$ \\
0.8 & 0.02020798979867 & 0.02020790518650 & $0.84612167911002 \mathrm{e}-7$ \\
0.9 & 0.01019799480033 & 0.01019790746566 & $0.87334672960529 \mathrm{e}-7$ \\
1 & 0.00018799980200 & 0.00018790974482 & $0.90057178009920 \mathrm{e}-7$ \\
\hline
\end{tabular}


Table 5 Comparisons between the exact solution and the approximate solution

\begin{tabular}{llll}
\hline $\boldsymbol{r}$ & $\underline{\boldsymbol{Y}(\boldsymbol{t}, \boldsymbol{r})}$ & $\underline{\boldsymbol{y}(\boldsymbol{t}, \boldsymbol{r})}$ & Error \\
\hline 0 & 2.00400200066667 & 2.00300328843835 & 0.00099871222831 \\
0.1 & 2.10430240100020 & 2.10320316558466 & 0.00109923541554 \\
0.2 & 2.20460280133373 & 2.20340304273096 & 0.00119975860278 \\
0.3 & 2.30490320166727 & 2.30360291987726 & 0.00130028179001 \\
0.4 & 2.40520360200080 & 2.40380279702356 & 0.00140080497724 \\
0.5 & 2.50550400233433 & 2.50400267416986 & 0.00150132816447 \\
0.6 & 2.60580440266787 & 2.60420255131616 & 0.00160185135171 \\
0.7 & 2.70610480300140 & 2.70440242846246 & 0.00170237453894 \\
0.8 & 2.80640520333493 & 2.80460230560876 & 0.00180289772617 \\
0.9 & 2.90670560366847 & 2.90480218275507 & 0.00190342091340 \\
1 & 3.00700600400200 & 3.00500205990137 & 0.00200394410063 \\
\hline
\end{tabular}

The exact solution of the equation is

$$
\begin{aligned}
& \underline{Y}(t, r)=(1+r) e^{2 t}+(-1+r) t e^{2 t}+t \\
& \bar{Y}(t, r)=(2-r) e^{2 t}+(1-r) t e^{2 t}+t
\end{aligned}
$$

The extended linear equations $S(t) X(r)=Y(r)$ is as follows:

$$
\begin{aligned}
\left(\begin{array}{cccccccc}
4 & 4 t & 2+4 t^{2} & 6 t+4 t^{3} & 0 & 4 & 8 t & 12 t^{2} \\
0 & 1 & 0 & 0 & 0 & 0 & 0 & 0 \\
0 & 1 & 2 & 0 & 0 & 0 & 0 & 0 \\
0 & 4 & 8 t & 12 t^{2} & 4 & 4 t & 2+4 t^{2} & 6 t+4 t^{3} \\
0 & 0 & 0 & 0 & 0 & 1 & 0 & 0 \\
0 & 0 & 0 & 0 & 0 & 1 & 2 & 0
\end{array}\right)\left(\begin{array}{l}
\underline{\alpha}_{0}(r) \\
\underline{\alpha}_{1}(r) \\
\underline{\alpha}_{2}(r) \\
\underline{\alpha}_{3}(r) \\
\bar{\alpha}_{0}(r) \\
\bar{\alpha}_{1}(r) \\
\bar{\alpha}_{2}(r) \\
\bar{\alpha}_{3}(r)
\end{array}\right) \\
=\left(\begin{array}{c}
4 t-4 \\
3+2 r \\
(-1+5 r) e^{2}+1 \\
4 t-4 \\
6-5 r \\
(7-5 r) e^{2}+1
\end{array}\right) .
\end{aligned}
$$

By setting $t=\frac{1}{6}$, the parameters $\underline{\alpha}_{0}(r), \underline{\alpha}_{1}(r), \underline{\alpha}_{2}(r), \underline{\alpha}_{3}(r), \bar{\alpha}_{0}(r), \bar{\alpha}_{1}(r), \bar{\alpha}_{2}(r), \bar{\alpha}_{3}(r)$ are obtained. Tables 5 and 6 show comparisons between the exact solution and the approximate solution at $t=0.001$ for some $r \in[0,1]$; all data were calculated by Matlab7.x.

Form Tables 1, 2, 3, 4, 5 and 6, we know that the approximate solutions obtained from the proposed method are best close to the exact solutions of original linear deferential equations with fuzzy boundary value conditions.

\section{Conclusion}

In this paper the approximate method similar to the undetermined coefficients method, based on a positive basis for solving second-order fuzzy linear boundary value problems, was discussed. Three classes of boundary conditions and the general case were considered. According to the sign of coefficient functions of the fuzzy linear differential equation, the 
Table 6 Comparisons between the exact solution and the approximate solution

\begin{tabular}{llll}
\hline $\boldsymbol{r}$ & $\overline{\boldsymbol{Y}}(\boldsymbol{t}, \boldsymbol{r})$ & $\overline{\boldsymbol{y}}(\boldsymbol{t}, \boldsymbol{r})$ & Error \\
\hline 0 & 4.01001000733734 & 4.008999990996165 & 0.00101009737569 \\
0.1 & 3.90970960700380 & 3.90880003281535 & 0.00090957418846 \\
0.2 & 3.80940920667027 & 3.80860015566904 & 0.00080905100122 \\
0.3 & 3.70910880633673 & 3.70840027852274 & 0.00070852781399 \\
0.4 & 3.60880840600320 & 3.60820040137644 & 0.00060800462676 \\
0.5 & 3.50850800566967 & 3.50800052423014 & 0.00050748143953 \\
0.6 & 3.40820760533613 & 3.40780064708384 & 0.00040695825230 \\
0.7 & 3.30790720500260 & 3.30760076993754 & 0.00030643506506 \\
0.8 & 3.20760680466907 & 3.20740089279124 & 0.00020591187783 \\
0.9 & 3.10730640433553 & 3.10720101564493 & 0.00010538869060 \\
1 & 3.00700600400200 & 3.00700113849863 & 0.00000486550337 \\
\hline
\end{tabular}

corresponding function systems of linear equations were set up. Following each other, fuzzy approximate solutions were obtained by solving a crisp function extended system of linear equations. Numerical examples show that our methods are practical and efficient.

\section{Competing interests}

The authors declare that they have no competing interests.

Authors' contributions

All authors contributed equally and significantly in writing this paper. All authors read and approved the final manuscript.

\section{Author details}

${ }^{1}$ College of Mathematics and Statistics, Northwest Normal University, Lanzhou, 730070, China. ${ }^{2}$ Department of Public Courses, Gansu College of Traditional Chinese Medicine, Lanzhou, 730000, China. ${ }^{3}$ College of Chemistry and Chemical Engineering, Northwest Normal University, Lanzhou, 730070, China.

\section{Acknowledgements}

The work is supported by the Natural Scientific Funds of PR China $(71061013,21175108)$ and the Youth Research Ability Project of Northwest Normal University (NWNU-LKQN-1120)

Received: 21 January 2013 Accepted: 2 August 2013 Published: 30 September 2013

\section{References}

1. Zadeh, LA: The concept of a linguistic variable and its application to approximate reasoning. Inf. Sci. 8, 199-249 (1975)

2. Dubois, D, Prade, H: Operations on fuzzy numbers. Int. J. Syst. Sci. 9, 613-626 (1978)

3. Puri, ML, Ralescu, DA: Differentials for fuzzy functions. J. Math. Anal. Appl. 91, 552-558 (1983)

4. Goetschel, R, Voxman, W: Elementary calculus. Fuzzy Sets Syst. 18, 31-43 (1986)

5. Kandel, A: Fuzzy dynamic systems and the nature of their solutions. In: Wang, PP, Chang, SK (eds.) Fuzzy Sets Theory and Application to Policy Analysis and Information Systems, pp. 93-1221. Plenum Press, New York (1980)

6. Kandel, A, Byatt, WJ: Fuzzy differential equations. In: Proceedings of the International Conference on Cybernetics and Society, Tokyo, pp. 1213-1216 (1987)

7. Abbasbandy, S, Allahviranloo, T: Numerical solutions of fuzzy differential equations by Taylor method. Comput. Methods Appl. Math. 2, 113-124 (2002)

8. Abbasbandy, S, Allahviranloo, T, Lopez-Pouso, O, Otadi, M: Numerical methods for fuzzy differential inclusions. Comput. Math. Appl. 48, 1633-1641 (2004)

9. Allahviranloo, T, Ahmady, N, Ahmady, E: Numerical solutions of fuzzy differential equations by predictor-corrector method. Inf. Sci. 177, 1633-1647 (2007)

10. Buckley, JJ, Feuring, T: Fuzzy initial value problem for Nth-order fuzzy differential equations. Fuzzy Sets Syst. 121 , 247-255 (2001)

11. Allahviranloo, T: Nth-order fuzzy linear differential equations. Inf. Sci. 178, 1309-1324 (2008)

12. O'Regan, D, Lakshkantham, V, Nieto, JJ: Initial and boundary value problems for fuzzy differential equations. Nonlinear Anal. 54, 405-415 (2003)

13. Lakshkantham, $\vee$, Murty, KN, Turner, J: Two points boundary value problems associated with nonlinear fuzzy differential equations. Math. Inequal. Appl. 4, 527-533 (2003)

14. Chen, M, Wu, C, Xue, X: On fuzzy boundary value problems. Inf. Sci. 178, 1877-1892 (2008)

15. Wu, CX, Ma, M: Embedding problem of fuzzy number space: Part I. Fuzzy Sets Syst. 44, 33-38 (1991)

16. Wu, CX, Ma, M: Embedding problem of fuzzy number space: Part III. Fuzzy Sets Syst. 46, 281-286 (1992)

17. He, O, Yi, W: On fuzzy differential equations. Fuzzy Sets Syst. 24, 321-325 (1989)

18. Bede, B: Note on "Numerical solutions of fuzzy differential equations by predictor-corrector method". Inf. Sci. 178, 1917-1922 (2008)

19. Bede, B, Gal, S: Generalizations of the differentiability of fuzzy-number-valued functions with applications to fuzzy differential equation. Fuzzy Sets Syst. 151, 581-599 (2005)

20. Ben-Israel, A, Greville, TNE: Generalized Inverses: Theory and Applications, 2nd edn. Springer, New York (2003) 
doi:10.1186/1687-2770-2013-212

Cite this article as: Guo et al.: Fuzzy approximate solutions of second-order fuzzy linear boundary value problems. Boundary Value Problems 2013 2013:212.

Submit your manuscript to a SpringerOpen ${ }^{\circ}$ journal and benefit from:

- Convenient online submission

Rigorous peer review

- Immediate publication on acceptance

- Open access: articles freely available online

- High visibility within the field

- Retaining the copyright to your article

Submit your next manuscript at $\boldsymbol{s p r i n g e r o p e n . c o m ~}$ 